small sample size meant that results did not reach statistical significance.

This study demonstrates discordance between clinical and biomarker findings regarding treatment efficacy in patients with HER2 FISH-positive breast cancer. Continued cellular proliferation was present in the majority of these patients despite clinical response to ongoing treatment with letrozole or tamoxifen, and this may be an indication of the potential for later resistance to therapy.

Original article Ellis MJ et al. (2006) Estrogen-independent proliferation is present in estrogen-receptor HER2-positive primary breast cancer after neoadjuvant letrozole. J Clin Oncol 24: 3019-3025

\section{Primary bone lymphoma: "excellent" prognosis following chemoradiotherapy}

Data on the optimum management and longterm prognosis of primary bone lymphoma are limited, especially with regard to moremodern treatment modalities using combined chemotherapy and radiotherapy. To address this issue, Beal and colleagues investigated all cases of this relatively rare condition treated at their center (the Memorial Sloan-Kettering Cancer Center) over a 40-year period.

The study analyzed treatment regimen, survival, and prognostic factors for a total of 82 patients (median age 48 years, range 1183 years) with primary bone lymphoma. The majority (80\%) presented with diffuse largecell lymphoma, and of these $81 \%$ had Ann Arbor stage I or II disease. Treatment regimens used were combined-modality therapy $(57 \%$ of patients), radiation therapy alone (14\% of patients), and chemotherapy alone (30\% of patients). After a median follow-up of 67 months (range 2-280 months), 5-year overall survival (OS), cause-specific survival (CSS), and freedom from treatment failure (FFTF) were $88 \%$, 96\%, and $81 \%$, respectively. Fiveyear OS and FFTF were significantly higher for patients treated with combined-modality versus single-modality therapy (95\% vs $78 \%$, $P=0.013$, and $90 \%$ vs $67 \%, P=0.025$, respectively). Favorable prognostic factors for OS, CSS and FFTF were age below 40 years and use of combined-modality therapy.

The data from this cohort of patients support the use of combined radiotherapy and chemotherapy in patients with primary lymphoma involving the bone. Using this modality prognosis is extremely good, with CSS rates of $96 \%$.

Original article Beal K et al. (2006) Primary bone

lymphoma: treatment results and prognostic factors with

long-term follow-up of 82 patients. Cancer 106: 2652-2656

\section{Perioperative chemotherapy prolongs survival in resectable gastric cancer}

It has long been known that chemotherapy prolongs survival of patients with inoperable gastric cancer. A new, multinational study has now shown that perioperative chemotherapy can prolong survival of patients with surgically resected gastric cancer, compared with patients treated with surgery alone. Benefits of perioperative chemotherapy might, however, depend on the regime chosen.

Cunningham et al. randomly allocated patients diagnosed with resectable gastric, gastroesophageal junction, or lower-esophageal adenocarcinoma to undergo either surgery alone $(n=253)$, or surgery plus three preoperative and three postoperative cycles of chemotherapy $(n=250)$. Chemotherapy cycles comprised $50 \mathrm{mg} / \mathrm{m}^{2}$ epirubicin and $60 \mathrm{mg} / \mathrm{m}^{2}$ cisplatin on day 1 , with continuous infusion of fluorouracil $200 \mathrm{mg} / \mathrm{m}^{2}$ daily for 21 days. Dose modification was permitted in response to treatment-related toxicity. Median follow up was 47-49 months.

Compared with patients who underwent surgery alone, patients who received perioperative chemotherapy had markedly improved progression-free survival (difference between groups, $P<0.001)$ and overall survival $(5$-year survival rates of $36 \%$ versus $23 \%$ ). As expected, they also had reduced tumor size and stage at surgery. Only 104 of 250 patients assigned to perioperative chemotherapy completed all six cycles; reasons for not completing chemotherapy varied, but patient choice was an important factor. The authors note that acceptability of this chemotherapy regime is impaired by the need for lengthy intravenous infusions, and suggest that substitution of newer agents such as capecitabine and oxaliplatin might improve patient compliance.

Original article Cunningham D et al. (2006) Perioperative chemotherapy versus surgery alone for resectable esophageal cancer. N Engl J Med 355: 11-20 